\title{
DUNHAM JACKSON \\ 1888-1946
}

\author{
WILLIAM L. HART
}

1. Introduction. My first meeting with Dunham Jackson occurred in September, 1916, at Cambridge, Massachusetts, on the day when I arrived to begin an interesting period as a Benjamin Peirce Instructor in the Department of Mathematics at Harvard University. At that time, Jackson was one of the youngest permanent members of Harvard's mathematical staff. I recall that he immediately made me feel as if we were old friends, and I never lost that reaction to him. Later, he and I served together briefly in the United States Army, and thereafter we were colleagues in the Department of Mathematics at the University of Minnesota from 1919 until his death. I am confident that my warm personal feelings for the man were typical of those held by all members of the Society who knew him intimately.

2. Biographical remarks. Dunham Jackson was born on July 24, 1888, son of William Dunham Jackson and Mary Vose Jackson, in Bridgewater, Massachusetts, where his father was a teacher in a normal school. He attended the elementary and secondary schools in Bridgewater and entered Harvard University in 1904, where he obtained his bachelor's degree in 1908 and his master's degree in mathematics in 1909. At the various stages in his academic training at Harvard University, he received all possible honors and scholarships due to the excellence of his work. In 1909, he was awarded a Sheldon Fellowship from Harvard University for graduate study in Europe, where he attended the University of Göttingen in 1909-1911, and the University of Bonn, briefly, in 1911. He received his Ph.D. in mathematics at Göttingen in 1911, with Professor Landau as his adviser. He returned to Harvard University as an instructor in mathematics in 1911 and became an assistant professor there in 1916. He performed valuable mathematical work for the U. S. Army during 1918-1919 as a captain in the Ordnance Department, where he served in the Ballistic Unit organized in Washington, D. C. by Dr. F. R. Moulton (then a major in the Ordnance Department). On June 20, 1918, Jackson married Harriet Spratt Hulley, whom he had met in 1917 while she was a graduate student in English at Radcliffe College. They had two daughters, Anne Hulley and Mary Eloise (Mrs. William J. Thorpe). He went to the University of Minnesota as a professor of mathematics in 1919 and held that position until his death on 
November 6, 1946, at the age of 58.

Jackson experienced a serious heart attack early in 1940. Thereafter, although he was able to return to duty for various periods at the University of Minnesota, his health steadily deteriorated. In 1940 , and after 1943, due to recurring illness he was frequently confined for long periods to his room either at home or in a hospital, and this was necessary continuously during the last 18 months of his life. Nevertheless, during his last year, he gave approval to four Ph.D. dissertations, the frnal one only two weeks before his death; also, a research paper which he finished in 1946 has been published posthumously. It was evident that, in spite of his long and trying illness, he maintained creative ability, interest in the progress of his advanced students and the affairs of the Society, and normal reactions to his family and friends to the end of his life.

The special abilities possessed by Jackson and his many services to the field of mathematics were appropriately recognized during his lifetime. He was a member of the Council of the Society from 1918 to 1920 , Vice-President in 1921, and a member of the Transactions Editorial Committee from 1926 to 1931. He gave the colloquium lectures at the Summer Meeting of the Society in 1925. He was a charter member of the Mathematical Association of America, a member of its Board of Governors from 1923 to 1929, Vice-President in 1924 and 1925, and President in 1926; he was awarded the Chauvenet Prize of the Association for the period 1932-1934 in 1935 (see \$4). He was a Vice-President of the American Association for the Advancement of Science in 1927, and Secretary of Section A (Mathematics) of that Association for many years. He was a fellow of the American Academy of Arts and Sciences and a member of the $\mathrm{Na}$ tional Academy of Sciences. He always maintained lively interest in mathematical affairs at the secondary level, and frequently played an active role in joint actions of the fields of college and secondary mathematics.

3. Personal qualities. Jackson was a man of high ideals, extremely unselfish, and very conscious of his responsibilities, not only in strictly personal affairs but also with respect to his profession as an educator and his duties as a citizen. He was intensely devoted to the field of mathematics, had thought deeply of its place in advancing human welfare, and was fluent in expressing his convictions on such matters. As an outstanding characteristic, he enjoyed his associations with people. In particular, his congenial wife and family were a continuing source of pleasure and inspiration to him. It was typical of the man that, after relatively few contacts, he was likely to call any new 
acquaintance by his given name as a sincere implication of permanent regard. Until his final illness restricted his activities, he was a regular attendant at meetings of the Society, where his cheerful manner and cordiality, in addition to his active interest in the mathematical programs, made him a great asset. One of his major regrets during his last few years was his inability to attend the meetings of the Society to renew old friendships and to make new ones. He always preferred to have a substantial part of his teaching schedule at the undergraduate level because he enjoyed personal contacts with young people and the opportunity to influence them scientifically at an early age. His students will remember him as a skillful and inspiring teacher who also was a sincere friend. His former associates in various activities will recall him not only because of his scholarly. attainments and his ability as a counselor, but also because of his warm personality, his lively sense of humor, and the breadth of his general interests. In his untimely death, the field of mathematics experienced a very great loss.

4. Preliminary summary of Jackson's mathematical work. He presented his first paper to the Society in September, 1909, before going to Göttingen for his final graduate study; this paper [1] $]^{1}$ dealt with a topic in algebra, but practically his entire remaining research was in the field of analysis. His last paper [63] was presented to the Society posthumously on December 26, 1946. In all, he published approximately 75 mathematical papers involving novelty in the results, or in the methods and organization. He was the author of two books, his colloquium lectures [38] and a volume [58] in the series of Carus Monographs. Also, he published various articles of general mathematical interest intended for teachers at the secondary or college level, or for the general public. While he was in the Army, he wrote a textbook in pamphlet form for the Ordnance Department on the method of numerical integration as applied in exterior ballistics. Jackson was noted for his ability as an expositor of mathematics in his published work, in lectures, and in teaching at all levels. The excellence of his expository papers [42], [45], and [46], on series of orthogonal polynomials, orthogonal trigonometric sums, and the convergence of Fourier series, was the basis for the award to him of the Chauvenet Prize of the Mathematical Association of America for the period 1932-1934.

${ }^{1}$ Numbers in brackets refer to the bibliography of Jackson's publications at the end of the article. 
Jackson's choice of a subject for his Göttingen dissertation permanently channeled his main research into the field of approximation theory and orthogonal functions, which he enriched by many new results and outstanding expositions of fundamental content. In the preface to his colloquium lectures [38], he made the following remarks concerning this choice on his part.

"Guided partly by natural inclinations, perhaps, and partly by recollection of a course on methods of approximation which $I$ had taken with Professor Bôcher a few years earlier, I committed myself to one of the topics which Landau had proposed (for a thesis), an investigation of the degree of approximation with which a given continuous function can be represented by a polynomial of given degree. ${ }^{2}$ When I reported my choice he said meditatively ... Das ist ein schönes Thema, ich beneide Sie um das Thema . . . Nein, ich beneide Sie nicht, aber es ist ein wunderschönes Thema."

5. Miscellaneous research. Before discussing the nature of Jackson's contributions in his major field, it is pertinent to mention that his published papers exhibit a reasonable diversity of content in other directions. Thus, for a few years while he was a member of the faculty of Harvard University, and occasionally later, he turned his attention to boundary value problems. One extensive paper [9] dealt with an ordinary linear differential equation and associated irregular boundary conditions, where he established the divergence of corresponding expansions of functions in series of characteristic functions, in contrast to convergence for the case of regular conditions. He discussed a system of boundary conditions associated with an ordinary linear differential equation of degree $n$ and obtained a matrix condition for the system to be self-adjoint [12]. He applied the contour integral method for a boundary value problem to obtain the customary formula for the partial sum of a Fourier series [13].

In an early period, he also investigated certain aspects of the theory of functions of several complex variables. Thus, he gave conditions under which such a function is the quotient of two polynomials [10]. $\mathrm{He}$ introduced two definitions of a non-essential singularity for a function of several complex variables which led to the conclusion that, in the neighborhood of the singularity, except at certain points, the function is expressible as a quotient of analytic functions [11]. In a study of semi-analytic functions $f(x, y)$, he obtained a theorem concluding with an expression for $f(x, y)$ as a product of two functions of

2 In considering this topic, Jackson enlarged it to include approximation by trigonometric sums as well as by polynomials. 
the same type, one factor being a polynomial in one variable and continuous in the other, in the neighborhood of a zero of $f(x, y)$; also, he obtained an expression for $f(x, y)$ as a quotient of semi-analytic functions in the neighborhood of any singularity, of the types he defined, except at certain points [14].

During the last two-thirds of Jackson's life at the University of Minnesota, he developed considerable interest in mathematical statistics and related topics although, simultaneously, he remained active in his main field. He presented a very useful new definition of percentiles designed to improve their mathematical formulation [23]. He proposed a novel symmetric coefficient of correlation for several variables [28]. He discussed various features of the geometry of function space designed as an aid to the appreciation of certain features of the theory of correlation [32], [34], [38, Chap. V]. In this connection, his exposition of some elements of sampling theory [48], and his clear presentation of contacts between Pearson frequency functions and various types of orthogonal polynomials [58; Chap. VI and later] deserve special mention.

In 1921, Jackson published an interesting brief paper [21] on the Picard method for establishing the existence of a solution of a system $d y_{i} / d x=f_{i}\left(x, y_{1}, y_{2}, \cdots, y_{n}\right)$, with $y_{i}=b_{i}$ when $x=a$, for $i=1,2$, $\cdots, n$. By simply extending the functions $f_{i}$ outside their original region of definition $R$, he proved in a few words, under the usual hypotheses, that the approximations of the method converge to the unique solution as long as it remains in $R$, if $R$ is of reasonably convenient shape. This important point had been open to question previously.

6. Doctoral dissertation and early related investigations. Jackson's thesis [2] was never published except as a separate pamphlet in German. This relative inaccessibility, and the importance of the content make it appropriate to refer to some of the details of the thesis at this point. His primary result was as follows:

There exists an absolute constant $K_{1}$ with the following property: if $f(x)$ has the period $2 \pi$ and satisfies a Lipschitz condition, $\left|f\left(x_{1}\right)-f\left(x_{2}\right)\right|$ $\leqq \lambda\left|x_{1}-x_{2}\right|$, then, for every positive integer $n$, there exists a trigonometric sum $^{8} T_{n}(x)$ of order $n$ at most so that

$$
\left|f(x)-T_{n}(x)\right| \leqq K_{1} \lambda / n
$$

for all values of $x$, and thus $\max \left|f(x)-T_{n}(x)\right|=O(1 / n)$.

${ }^{8}$ Throughout the article, $T_{n}$ and $P_{n}$ will have the meanings indicated in this section. 
In his demonstration of this result, $T_{n}(x)$ was explicitly exhibited. Under similar hypotheses on a function $f(x)$ defined on an interval $(a, b)$, omitting reference to periodicity, he exhibited polynomials $P_{n}(x)$ of degree $n$ at most so that $\left|f(x)-P_{n}(x)\right| \leqq G_{1} \lambda l / n$, where $l=b-a$ and $G_{1}$ is an absolute constant. Moreover, if $T_{n}(x)$ now represents the trigonometric sum of order $n$ at most for which $\max \mid f(x)$ $-T_{n}(x) \mid \equiv \phi(n)$ has its least value, Jackson proved the important result that, under his hypotheses, in general $\phi(n)$ is not of lower order than $1 / n$, with a similar result for the polynomial case. If $f(x)$ possesses an $(h-1)$ th derivative satisfying a Lipschitz condition, Jackson obtained $K_{h} \lambda / n^{h}$ in place of the right-hand side of (1), and a parallel result for polynomials. He proved similar theorems for a function $f(x, y)$ with respect to approximation by polynomials $P_{n}(x, y)$. The key detail in Jackson's thesis was an expression for $f(x)$ by means of a new type of singular integral, probably suggested by those which had been employed previously in proofs of Weierstrass's theorem about polynomial approximation. Jackson's thesis answered a prize question which had been proposed earlier by the Göttingen faculty: "Would it be possible to improve on results (on approximation by polynomials) previously obtained by de la Vallee Poussin and Lebesgue?" In a complimentary statement awarding Jackson the prize, we find the remark: "The author ... has enriched the science with valuable results, all in competition with mathematicians of the first rank."

Jackson's thesis provided powerful assistance for studying the degree of convergence of Fourier series, or series in orthogonal polynomials. After returning to the United States from Germany, he quickly used these new tools. First, he discussed the degree of convergence of Legendre series [3]. Then, he published considerably simplified proofs of those results of his thesis concerning upper bounds of the order of approximation; he obtained close lower bounds for the important absolute constants, such as $K_{1}$ in (1); he investigated the degree of convergence of Fourier series under various hypotheses [4], [5]. He extended his methods to a study of approximation by trigonometric interpolation formulas [6], [7], and rounded out this theory six years later [15]. In the direction of boundary value problems, he obtained results, similar to those for Fourier series, on the degree of convergence of an interesting class of Sturm-Liouville series [8]. He gave a simple proof of a Fejer theorem on the uniform summability, in the sense of the first arithmetic mean, of the Fourier series for a periodic continuous function $f(x)$ [16]. Jackson's method employed the Weierstrass theorem on the approximation of $f(x)$ by means of trigonometric sums, and a modification of a standard meth- 
od which had been used in discussing the ordinary convergence of Fourier series.

7. Approximation in the sense of least $m$ th powers; $1921-1925$. A new stage of Jackson's main work was initiated by his pioneer investigations of "best" approximating trigonometric sums $T_{n, m}(x)$, or polynomials $P_{n, m}(x)$, where the measure of the error (stating it for the trigonometric case) of $T_{n, m}(x)$ as an approximation to $f(x)$ was taken as $\int_{0}^{2 x}\left|f(x)-T_{n, m}(x)\right|^{m} d x$, with fixed $m>0.4$ The objective here was to generalize the familiar least-square case $(m=2)$, where $T_{n, m}$ becomes the $n$th partial sum of the Fourier series, and $P_{n, m}$ is the $n$th partial sum of the Legendre series for $f(x)$. In a group of papers $[17],[18],[20],[25]$ he established the existence of such best approximations $T_{n, m}$, or $P_{n, m}$, and their convergence to $f(x)$ as $n$ becomes infinite, under the various assumptions $m \geqq 1$ (unique determination of $T_{n, m}$ and $P_{n, m}$ ) and $0<m<1$ (possibly ambiguous case). His treatment was phrased for a family of approximating functions including, as special cases, the family of all trigonometric sums, or all polynomials. An interesting by-product was his proof that $P_{n, m}(x)$ tends to the Tchebichef polynomial of degree $n$ as $m$ becomes infinite [17]. Then, he widened the scope of the treatment by the introduction of a non-negative weight function $\rho(x)$ as a factor under the integral sign in the expression for the measure of error [24], [27], as found in earlier literature for the case $m=2$.

In connection with the research just mentioned, we observe various related investigations by Jackson. He applied a method of "least $m$ th powers" in the solution of a system of linear equations [29]. He gave a treatment of approximation with a general function $E(y)$, subject only to liberal hypotheses, in place of $|y|^{m}$ as the essential analytic feature of the integrand in the expression for the error of approximation [30]. Also, he considered trigonometric approximation in the sense of least $m$ th powers, without a weight function, to an "ill-defined" function $y=f(x)$, where the many-valued function $y$ has values distributed (statistically) in accordance with a frequency function $w(x, y)$; he reduced this problem to one of ordinary type for a new single-valued function $F(x)$, to be approximated in the sense of least $m$ th powers with an appropriate weight function [31].

8. Comprehensive research reports to the Society. In 1921, at a symposium of the Society in Chicago, and at the Summer meeting

4 The polynomial case had previously been treated to a certain extent by Polya in a note in C. R. Acad. Sci. Paris vol. 157 (1913) pp. 840-843. Jackson's original work in this direction was done without knowledge of P6lya's note. 
of the Society in 1925, Jackson had opportunities to present extensive discussions concerning his major field. His symposium lecture was essentially a synoptic treatment of his own main research up to 1921 and related literature [19]. His colloquium lectures of 1925 on approximation theory, as published in 1930 [38], include complete presentations of essential parts of his own research, associated new results and refinements of existing literature, and collateral classical material to give a rounded view of the field. A final chapter, loosely connected with the other content through the notions of least-square approximation and orthogonality, deals with the geometry of function space. This chapter leads to geometrical viewpoints about certain features of correlation theory in statistics, and had been published previously by Jackson.

9. Expository and detached publications in his main field; 19261946. Jackson's mathematical publications after 1925 involved several excursions (already mentioned) into the theory of statistics, particularly before 1930. In his field of major interest, he devoted considerable time to expositions of fundamental content, written at the level of readers satisfying only minimum mathematical prerequisites, in order to make the material more accessible in its fields of application. His principal work of this type was his Carus Monograph [58], 1941, on Fourier series and orthogonal polynomials, which requires only a substantial first course in calculus, and active interest, as the prerequisites for intelligent appreciation of most of the content. His other outstanding expository publications are the three related papers (loc. cit.) for which he was awarded the Chauvenet Prize in 1935. There is considerable overlapping between these papers and his Carus Monograph.

In this period, Jackson published various results somewhat apart from his main program (which I will mention later). Thus, I first note a few papers on problems of approximation in the complex domain. In [37], Jackson discussed the existence of $P_{n}(z)$ to minimize $\int_{C} \rho(s)\left|f(z)-P_{n}(z)\right|^{m} d s$, where $C$ is the boundary of a closed region $S$, and considered the convergence of $P_{n}(z)$ to $f(z)$ in $S ; C$ was subject to restrictions designed to permit the application of Bernstein's theorem on the maximum of the derivative of a polynomial in z. This gave results similar to some previously obtained otherwise by J. L. Walsh, cited in [37]. In [40], Jackson relaxed the preceding condition on $C$ and, in his proofs, replaced Bernstein's theorem by one of Markoff, with somewhat more stringent hypotheses about $f(z)$. In [26] at an earlier period, [36], and [43], Jackson considered vari- 
ous "nonlinear" problems of approximation (returning to the real domain), where in each case the approximating functions belong to a nonlinear family. He investigated problems where the approximating functions are required to satisfy auxiliary conditions, point-wise [33], [35], or expressed by means of integrals [41].

10. Foundation papers for a major program. The remaining research by Jackson to which I wish to refer appeared in 1933-1946, and is roughly connected in regard to methods and viewpoints. These publications start with four papers (two are expository) which establish patterns for later proofs and concentrate essential reference material from many sources in a few convenient locations. Thus, in his expositions concerning systems of polynomials [42] and trigonometric sums [45] orthonormal with respect to a weight function $\rho(x)$, he emphasized the role played by the property of boundedness for the orthonormal functions, in conjunction with the corresponding Christoffel-Darboux identity, in proving the convergence of related expansions. In a third paper [44], he obtained various upper bounds for the order of magnitude of $\left|P_{n}(x)\right|$ as a function of $n$ in case $P_{n}(x)$ is normalized, $\int_{a}^{b} \rho(x)\left|P_{n}(x)\right|^{2} d x=1$. Also, as an independent part of [44], if $P_{n}$ minimizes $\int_{a}^{b} \rho(x)\left|f(x)-P_{n}(x)\right|^{\circ} d x$, he developed standard devices for establishing an upper bound for the error of approximation, $\left|f(x)-P_{n}(x)\right|$, in terms of an upper bound, $\epsilon_{n}$, of the error of approximation to $f(x)$ attainable by use of other polynomials of degree $n$ at most. The hypotheses of theorems on the convergence of $P_{n}(x)$ to $f(x)$ are then stated in terms relating to the rapidity with which $\epsilon_{n}$ tends to zero as $n$ becomes infinite, where such facts are considered as known under various hypotheses about $f(x)$ from Jackson's earliest research. The key detail of [44] is an expression for an upper bound of $\left|P_{n}(x)\right|$ in terms of the value of $\int_{a}^{b}|P(x)|^{d} d x$, and the proofs make frequent use of Bernstein's and Markoff's theorems on the magnitude of the derivative of $P_{n}(x)$. Parallel results are developed in [44] for trigonometric sums $T_{n}(x)$, with integration from $x=0$ to $x=2 \pi$. In a fourth foundation paper [49], he extended the preceding results with respect to $T_{n}$ to the inconvenient case where all integration involved is from $x=c$ to $x=d$, with $0<c<d<2 \pi$. In convergence results, [44] and [49] are noteworthy because of the relative liberality of the hypotheses with regard to the troublesome feature of the vanishing of the weight function $\rho(x)$.

Adaptations of methods from some of the preceding papers were used by Jackson to obtain theorems on the summability of expansions in series of orthonormal polynomials or trigonometric sums [47]. 
Also, in [53], he applied methods like those of [44] and [49] to the two-dimensional case, where he considered approximation to $f(x, y)$ over a region $R$ by use of polynomials $P_{n}(x, y)$ or trigonometric sums $T_{n}(x, y)$. In this discussion, an essential preliminary detail was the development of analogs, for two dimensions, of the standard Bernstein and Markoff theorems; Jackson obtained these generalizations on the basis of a moderate restriction on the region $R$.

11. Orthonormal polynomials in two or more variables; orthonormal systems on algebraic loci; 1936-1946. In a sequence of publications commencing with [50] in 1936 and ending with Jackson's final research paper, he concerned himself with systems of polynomials (and, in a few instances, trigonometric sums) in two or three variables which are orthogonal and normalized, for a given weight function $\rho$, with respect to integration over a specified closed point set $R$ of one, two, or three dimensions. In [50], he considered the system of polynomials $\left[p_{n k}(x, y)\right]$, where $p_{n k}$ is of degree $n$ and $n=1,2, \cdots$, which are orthonormal over a region $R$; the orthonormality condition is that $\iint_{R} \rho(x, y) p_{n k}(x, y) p_{g h}(x, y) d x$ equals 1 or zero according as $|n-g|+|k-h|$ equals zero, or is not equal to zero. For this system, he developed a recursion formula and an analog of the ChristoffelDarboux identity, and discussed features arising under transformation of the variables. He paralleled these considerations in [55] for a system $\left[p_{n k}(x, y, z)\right]$, orthonormal with respect to integration over a point set $R$ which might be a region, a surface, or a curve. If a function $f$, defined in $R$, is expanded formally in a series of such polynomials, he sketched least-square reasoning in various situations to prove that the series converges to $f$ in the mean, weighted by $\rho$, if suitable restrictions are placed on $\rho$ and the given function [55, p. 445]. He then turned his attention primarily to cases of the interesting type where a system $\left[p_{n k}\right]$ is orthonormal on a locus which is algebraic. The characteristic feature here is that, due to whatever algebraic identity or identities may be associated with the locus, the number $h_{n}$ of polynomials of degree $n$ in the orthonormal system is smaller than for a general system. With each type of algebraic locus which he considered later, a discussion of the order of magnitude of $h_{n}$ was an essential detail. Thus, for an algebraic plane curve, he found that there exists a constant $N$ so that $h_{n}=N$, if $n \geqq N$ [51]. He discussed $h_{n}$ and formal properties of the orthonormal system for an algebraic plane curve [51], and for an algebraic surface or an algebraic space curve [55].

On the basis of results just mentioned, Jackson investigated the 
convergence of the expansion of a function $f(x, y)$, defined on an algebraic plane curve $C$, in series of polynomials $p_{n k}(x, y)$ orthonormal with respect to integration on $C$. In a group of papers, his apparent object was to illustrate characteristic difficulties, methods, and results by means of special examples, since a general treatment appeared to be an unfruitful venture. One of his methods was to develop a modification of Bernstein's theorem applicable on the special curve $C$ involved; then, he could conclude by merely remarking that standard methods of the foundation paper [44] applied in treating convergence. As another method, Jackson emphasized the classical use of the Christoffel-Darboux identity, obtained in [51], to establish convergence in case $p_{n k}(x, y)$ is uniformly bounded, for all $n$ and $k$. As an aid in proving this property for specific cases, he established a theorem, in [59], stating hypotheses on $C$ and a function $\rho(x, y)$ so that, if the orthonormal polynomials $p_{n k}(x, y)$ on $C$ are known to be uniformly bounded for a given weight function $\sigma(x, y)$, we may infer that the orthonormal polynomials $q_{n k}(x, y)$ for the new weight function $\rho \sigma$ also will be uniformly bounded (generalization of a theorem of Korous). Then, Jackson's procedure with a special curve $C$ was to produce one weight function $\sigma(x, y)$, sometimes $\sigma \equiv 1$, for which the polynomials $p_{n k}(x, y)$ were uniformly bounded. From this, he would then conclude that a similar boundedness property, and hence convergence of expansions, held for a wide class of weight functions of the type $\rho \sigma$. With these remarks as to Jackson's methods, his papers [52 ], [60], [62], [63] on orthonormal polynomials on special algebraic curves are sufficiently described by reading their titles in the bibliography. Special results of a related character, under a less descriptive title, are given in [61] for certain curves of rational type in two and three dimensions. In regard to polynomials $p_{n k}(x, y, z)$ orthonormal on an algebraic surface $R$, Jackson treated the convergence of the associated expansion of a function $f(x, y, z)$ only for one illustration, where $R$ is the unit sphere and the weight function is unity [55].

Jackson also considered notions related to the preceding content, extended in two directions. In [54], he discussed a system of orthonormal polynomials $\left[p_{n k}\left(z_{1}, z_{2}\right)\right]$ of the complex variables $z_{1}$ and $z_{2}$, where the relation of orthogonality is of the form

$$
\int_{E} \rho\left(z_{1}, z_{2}\right) p_{n k}\left(z_{1}, z_{2}\right) p_{o h}\left(z_{1}, z_{2}\right) d E=0, \quad|n-g|+|k-\not| \neq 0,
$$

in which $\rho \geqq 0$, and $E$ is a one-, two-, three-, or four-dimensional spread in the space of the corresponding four real coordinates $\left(x_{1}, y_{1}, x_{2}, y_{2}\right)$. In regard to the expansion of a function $f\left(z_{1}, z_{2}\right)$ in series of the polynomials $p_{n k}\left(z_{1}, z_{2}\right)$, he treated convergence in the mean for any range 
$E$, and ordinary convergence for illustrative simple ranges $E$. In [56], he investigated systems of "mixed sums" $u_{k}(x, y)$, orthonormal with respect to integration on a plane curve $C$, where $u_{k}(x, y)$ is a trigonometric sum in $x$ with coefficients which are polynomials in $y$. Also, in [56] he touched lightly on systems where $u_{k}$ is a trigonometric sum in each variable. To illustrate possibilities, he discussed the convergence of expansions in series of mixed sums $u_{k}$ for a special curve $C$ defined by an identity which involves a mixed sum.

12. Conclusion. A discussion of the mathematical work of any member of a graduate faculty is incomplete without comment on his activities in connection with doctoral dissertations. Jackson directed the thesis research of eighteen successful candidates for the Ph.D. degree at the University of Minnesota, and of two candidates at Harvard University during the first few years of his career as a teacher. The topics of these theses, as is to be expected, present a cross section of his research interests during the corresponding periods.

\section{BIBLIOGRAPHY}

The following partial list of Jackson's publications includes only those referred to in the preceding article.

1. Resolution into involutory substitutions of the transformations of a non-singular bilinear form into itself, Trans. Amer. Math. Soc. vol. 10 (1909) pp. 479-484.

2. Über die Genauigkeit der Annaherung stetiger Funktionen durch ganze rationale Funktionen gegebenen Grades und trigonometrische Summen gegebener Ordnung, Gekrönte Preisschrift und Inaugural-Dissertation, Göttingen University, 1911, pamphlet, $98 \mathrm{pp}$.

3. On the degree of convergence of the development of a continuous function according to Legendre polynomials, Trans. Amer. Math. Soc. vol. 13 (1912) pp. 305-318.

4. On approximation by trigonometric sums and polynomials, Trans. Amer. Math. Soc. vol. 13 (1912) pp. 491-515.

5. On the approximate representation of an indefinite integral and the degree of convergence of related Fourier series, Trans. Amer. Math. Soc. vol. 14 (1913) pp. 343-364.

6. On the accuracy of trigonometric interpolation, Trans. Amer. Math. Soc. vol. 14 (1913) pp. 453-461.

7. A formula of trigonometric interpolation, Rend. Circ. Mat. Palermo vol. 37 (1914) pp. 371-375.

8. On the degree of convergence of Sturm-Liouville series, Trans. Amer. Math. Soc. vol. 15 (1914) pp. 439-466.

9. Expansion problems with irregular boundary conditions, Proceedings of the American Academy of Arts and Sciences vol. 51 (1915) pp. 381-417.

10. Note on rational functions of several complex variables, J. Reine Angew. Math. vol. 146 (1916) pp. 185-188.

11. Non-essential singularities of functions of several complex variables, Ann. of Math. (2) vol. 17 (1916) pp. 172-179.

12. Algebraic properties of self-adjoint systems, Trans. Amer. Math. Soc. vol. 17 (1916) pp. 418-424. 
13. Note on representations of the partial sum of a Fourier series, Ann. of Math. (2) vol. 18 (1917) pp. 139-146.

14. Roots and singular points of semi-analytic functions, Ann. of Math. (2) vol. 19 (1917) pp. 142-151.

15. On the order of magnitude of coefficients in trigonometric interpolation, Trans. Amer. Math. Soc. vol. 21 (1920) pp. 321-332.

16. Note on a method of proof in the theory of Fourier series, Bull. Amer. Math. Soc. vol. 27 (1920) pp. 108-110.

17. On functions of closest approximation, Trans. Amer. Math. Soc. vol. 22 (1921) pp. 117-128.

18. On the convergence of certain trigonometric and polynomial approximations, Trans. Amer. Math. Soc. vol. 22 (1921) pp. 158-166.

19. The general theory of approximation by polynomials and trigonometric sums, Bull. Amer. Math. Soc. vol. 27 (1921) pp. 415-431.

20. Note on a class of polynomials of approximation, Trans. Amer. Math. Soc. vol. 22 (1921) pp. 320-326.

21. Note on the Picard method of successive approximations, Ann. of Math. (2) vol. 23 (1921) pp. 75-77.

22. Note on an irregular expansion problem, Bull. Amer. Math. Soc. vol. 28 (1922) pp. 37-41.

23. Note on quartiles and allied measures, Bull. Amer. Math. Soc. vol. 29 (1923) pp. 17-20.

24. Note on convergence of weighted trigonometric series, Bull. Amer. Math. Soc. vol. 29 (1923) pp. 259-263.

25. Note on an ambiguous case of approximation, Trans. Amer. Math. Soc. vol. 25 (1923) pp. 333-337.

26. On approximation by functions of given continuity, Trans. Amer. Math. Soc. vol. 25 (1923) pp. 449-458.

27. A general problem in weighted approximation, Trans. Amer. Math. Soc. vol. 26 (1924) pp. 133-154.

28. A symmetric coefficient of correlation for several variables, Bull. Amer. Math. Soc. vol. 30 (1924) pp. 536-542.

29. On the method of least mth powers for a set of simultaneous equations, Ann. of Math. (2) vol. 25 (1924) pp. 185-192.

30. A general class of problems in approximation, Amer. J. Math. vol. 46 (1924) pp. 215-234.

31. On the trigonometric representation of an ill-defined function, Ann. of Math. (2) vol. 26 (1924) pp. 8-20.

32. The geometry of frequency functions, Bull. Amer. Math. Soc. vol. 31 (1925) pp. 63-73.

33. Note on a problem of approximation with auxiliary conditions, Bull. Amer. Math. Soc. vol. 32 (1926) pp. 259-262.

34. Some convergence proofs in the vector analysis of function space, Ann. of Math. (2) vol. 27 (1926) pp. 551-567.

35. On the approximate representation of analytic functions, Bull. Amer. Math. Soc. vol. 34 (1928) pp. 56-62.

36. Some non-linear problems in approximation, Trans. Amer. Math. Soc. vol. 30 (1928) pp. 621-629.

37. On certain problems of approximation in the complex domain, Bull. Amer. Math. Soc. vol. 36 (1930) pp. 851-857. 
38. The theory of approximation, Amer. Math. Soc. Colloquium Publications, vol. $11,1930,8+178 \mathrm{pp}$.

39. Note on the convergence of a sequence of approximating polynomials, Bull. Amer. Math. Soc. vol. 37 (1931) pp. 69-72.

40. On the application of Markoff's theorem to problems of approximation in the complex domain, Bull. Amer. Math. Soc. vol. 37 (1931) pp. 883-890.

41. Problems of approximation with integral auxiliary conditions, Amer. J. Math. vol. 55 (1933) pp. 153-166.

42. Series of orthogonal polynomials, Ann. of Math. (2) vol. 34 (1933) pp. 527-545.

43. The convergence of some non-linear processes of approximation, Amer. J. Math. vol. 55 (1933) pp. 515-524.

44. Certain problems of closest approximation, Bull. Amer. Math. Soc. vol. 39 (1933) pp. 889-906.

45. Orthogonal trigonometric sums, Ann. of Math. (2) vol. 34 (1933) pp. 799-814. 84.

46. The convergence of Fourier series, Amer. Math. Monthly vol. 41 (1934) pp. 67-

47. The summation of series of orthogonal polynomials, Bull, Amer. Math. Soc. vol. 40 (1934) pp. 743-752.

48. Mathematical principles in the theory of small samples, Amer. Math. Monthly vol. 42 (1935) pp. 344-364.

49. Bernstein's theorem and trigonometric approximation, Trans. Amer. Math. Soc. vol. 40 (1936) pp. 225-251.

50. Formal properties of orthogonal polynomials in two variables, Duke Math. J. vol. 2 (1936) pp. 423-434. 236.

51. Orthogonal polynomials on a plane curve, Duke Math. J. vol. 3 (1937) pp. 228-

52. Polynomial approximation on a curve of the fourth degree, Bull. Amer. Math. Soc. vol. 43 (1937) pp. 388-393.

53. Problems of closest approximation on a two-dimensional region, Amer. J. Math. vol. 60 (1938) pp. 436-446.

54. Orthogonal polynomials in two complex variables, Ann. of Math. (2) vol. 39 (1938) pp. 262-268. 454.

55. Orthogonal polynomials in three variables, Duke Math. J. vol. 4 (1938) pp. 441-

56. A class of orthogonal functions on plane curves, Ann. of Math. vol. 40 (1939) pp. $521-532$.

57. Orthogonal polynomials with auxiliary conditions, Trans. Amer. Math. Soc. vol. 48 (1940) pp. 72-81.

58. Fourier series and orthogonal polynomials, Carus Mathematical Monographs, No. 6, 1941, 8+234 pp.

59. Generalization of a theorem of Korous on the bounds of orthonormal polynomials, Bull. Amer. Math. Soc. vol. 48 (1942) pp. 602-608.

60. Boundedness of orthonormal polynomials on loci of the second degree, Duke Math. J. vol. 11 (1944) pp. 351-365.

61. The boundedness of certain sets of orthonormal polynomials in one, two, and three variables, Trans. Amer. Math. Soc. vol. 58 (1945) pp. 167-183.

62. The boundedness of orthonormal polynomials on certain curves of the third degree, Bull. Amer. Math. Soc. vol. 52 (1946) pp. 899-907.

63. The boundedness of orthonormal polynomials on certain curves of the fourth degree, Trans. Amer. Math. Soc. vol. 63 (1948) pp. 193-206. 